\title{
Differences in environmental stability among SARS-CoV-2 variants of concern: Omicron has higher stability
}

\section{Running title: Omicron has higher environmental stability}

\author{
Author list \\ Ryohei Hirose, ${ }^{1,2 *}$ Yoshito Itoh, ${ }^{2}$ Hiroshi Ikegaya ${ }^{3}$ Hajime Miyazaki, ${ }^{1,2}$ Naoto Watanabe, ${ }^{1,2}$ \\ Takuma Yoshida, ${ }^{1,2}$ Risa Bandou, ${ }^{1,3}$ Tomo Daidoji, ${ }^{1}$ Takaaki Nakaya ${ }^{1}$
}

\section{Author affiliations}

${ }^{1}$ Department of Infectious Diseases, Graduate School of Medical Science, Kyoto Prefectural University of Medicine, 465 Kajii-cho, Kawaramachi-Hirokoji, Kamigyo-ku, Kyoto 602-8566, Japan.

${ }^{2}$ Department of Molecular Gastroenterology and Hepatology, Graduate School of Medical Science, Kyoto Prefectural University of Medicine, 465 Kajii-cho, Kawaramachi-Hirokoji, Kamigyo-ku, Kyoto 602-8566, Japan.

${ }^{3}$ Department of Forensic Medicine, Graduate School of Medical Science, Kyoto Prefectural University of Medicine, 465 Kajii-cho, Kawaramachi-Hirokoji, Kamigyo-ku, Kyoto 602-8566, Japan.

*Corresponding author:

Ryohei Hirose 
Department of Infectious Diseases, Graduate School of Medical Science, Kyoto Prefectural University of Medicine, 465 Kajii-cho, Kawaramachi-Hirokoji, Kamigyo-ku, Kyoto 602-8566, Japan. Tel.: +81-75-251-5325, fax: +81-75-251-5328, e-mail: ryo-hiro@koto.kpu-m.ac.jp

\section{Keywords}

SARS-CoV-2; variants of concern; stability; disinfection effectiveness; human skin; survival time; infection control; contact transmission. 


\begin{abstract}
SARS-CoV-2 variants of concern (VOCs) could cause significant human and economic damage owing to increased infectivity and transmissibility, and understanding their characteristics is crucial for infection control. Here, we analyzed differences in viral stability and disinfection efficacy between the Wuhan strain and all VOCs. On plastic and skin surfaces, Alpha, Beta, Delta, and Omicron variants exhibited more than two-fold longer survival than the Wuhan strain, and the Omicron variant had the longest survival time. Specifically, survival times of the Wuhan strain, Alpha variant, Beta variant, Gamma variant, Delta variant, and Omicron variant on skin surfaces were 8.6 h (95\% CI, 6.5-10.9 h), 19.6 h (95\% CI, 14.8-25.3 h), 19.1 h (95\% CI, 13.9$25.3 \mathrm{~h}), 11.0 \mathrm{~h}(95 \% \mathrm{CI}, 8.1-14.7 \mathrm{~h}), 16.8 \mathrm{~h}(95 \% \mathrm{CI}, 13.1-21.1 \mathrm{~h})$, and $21.1 \mathrm{~h}(95 \% \mathrm{CI}, 15.8-$ $27.6 \mathrm{~h}$ ), respectively. In vitro, disinfectant effectiveness evaluations showed that Alpha, Beta, Delta, and Omicron were slightly more resistant to ethanol than the Wuhan strain. However, ex vivo evaluation showed that on human skin, all viruses were completely inactivated by exposure to $35 \mathrm{w} / \mathrm{w} \%$ ethanol for $15 \mathrm{~s}$. The high environmental stability of these VOCs could increase transmission risk and contribute to spread. Additionally, the Omicron variant might have been replaced by the Delta variant due to its increased environmental stability and rapid spread. To prevent VOC spread, it is highly recommended that current infection control practices use disinfectants with appropriate ethanol concentrations.
\end{abstract}




\section{Introduction}

Various SARS-CoV-2 variants have emerged from 2020 to 2021. In particular, SARS-CoV-2 variants classified as variants of concern (VOCs) can cause significant human and economic damage, and an understanding their characteristics is crucial for infection control. VOCs have been reported to have increased infectivity and transmissibility (1). In particular, the rapid spread of the Omicron (Pango lineage: B.1.1.529) variant has become a serious concern worldwide as of $2022(2,3)$. The increase in the infectivity/transmissibility can be attributed to several factors, such as increased viral load shed from infected individuals, prolonged viral shedding period, decrease in the minimum viral load required to establish infection, changes in infection target site, and increased environmental stability $(4,5)$.

The environmental stability of SARS-CoV-2 has been compared with that of other viruses, such as SARS-CoV-1 and influenza virus $(6,7)$. Moreover, previous studies have suggested that Alpha (Pango lineage: B.1.1.7) and Beta (Pango lineage: B.1.351) variants have the same degree of environmental stability $(8,9)$. However, the differences in viral stability among all VOCs, including the Omicron and Delta (Pango lineage: B.1.617.2) variant, have not been evaluated and compared in detail. Here, we improved our previously developed evaluation model and precisely analyzed the differences in viral stability and disinfection efficacy between the Wuhan strain (Pango lineage: A) and all VOCs. 


\section{Materials and methods}

\section{Viruses and cells}

The SARS-CoV-2 variants analyzed in this study were the Wuhan strain (Pango lineage: A, hCoV-19/Japan/TY/WK-521/2019), Alpha variant (Pango lineage: B.1.1.7, hCoV19/Japan/QK002/2020), Beta variant (Pango lineage: B.1.351, hCoV-19/Japan/TY8-612/2021), Gamma variant (Pango lineage: P.1, hCoV-19/Japan/TY7-501/2021), Delta variant (Pango lineage: B.1.617.2, hCoV-19/Japan/TY11-927/2021), and Omicron variant (Pango lineage: B.1.1.529, hCoV-19/Japan/TY38-873/2021). All viruses were generously provided by the National Institute of Infectious Diseases (Tokyo, Japan). For virus culture and quantification, VeroE6/TMPRSS2 cells, expressing the transmembrane serine protease TMPRSS2, were purchased from the Japanese Collection of Research Bioresources Cell Bank (Osaka, Japan) and cultured in Dulbecco's modified Eagle's medium (DMEM; Sigma Aldrich) supplemented with 5\% fetal bovine serum and G418 (Nacalai Tesque, Kyoto, Japan) $(10,11)$. The viruses were concentrated and purified as follows: $96 \mathrm{~h}$ post-infection, the culture medium was harvested and centrifuged for $10 \mathrm{~min}$ at $2,500 \times \mathrm{g}$ at $4{ }^{\circ} \mathrm{C}$ to eliminate cellular debris. Virions in the supernatant were sedimented through a $20 \%(\mathrm{w} / \mathrm{w})$ sucrose cushion in phosphate-buffered saline (PBS) via ultracentrifugation at $27,000 \mathrm{rpm}$ for $2.5 \mathrm{~h}$ at $4{ }^{\circ} \mathrm{C}$ using a Beckman SW28 rotor $(12,13)$. The titers of the virus were measured in terms of $50 \%$ tissue culture infectious dose (TCID 50$)$ in VeroE6/TMPRSS2 cells. Three days after inoculation, the cytopathic effect in each well was scored under a microscope, and the TCID 50 was calculated.

\section{Construction of skin model to evaluate virus stability and disinfectant effectiveness}


Human skin was collected from forensic autopsy specimens obtained from the Department of Forensic Medicine, Kyoto Prefectural University of Medicine. Abdominal skin autopsy specimens from subjects aged 20 to 70 years, obtained approximately $1 \mathrm{~d}$ after death, were cut into squares with approximate dimensions of $4 \mathrm{~cm} \times 8 \mathrm{~cm}$. Those whose skin was considerably damaged by burning or drowning were excluded. Using the skin-autopsy specimens, an ex vivo model was developed to evaluate the stability of different viruses on the surface of human skin and the effectiveness of different disinfectants against viruses on human skin $(6,14)$. The skin from which the panniculus adiposus that had been removed was washed with PBS and placed in a culture insert (Corning, Corning, NY, USA) on a membrane with a pore size of $8.0 \mu \mathrm{m}$. The culture inserts were placed in six-well plates containing 1.0 mL of DMEM (Sigma-Aldrich).

\section{Evaluation of virus stability on plastic and human skin surfaces}

Virus survival was evaluated on plastic (polystyrene plate) and human skin (constructed skin model) surfaces. Virus solutions $\left(5.0 \times 10^{4} \mathrm{TCID}_{50}\right.$ in $\left.2 \mu \mathrm{L} \mathrm{PBS}\right)$ were applied on the surface of plastic or human skin. Each sample was incubated in a controlled environment $\left(25{ }^{\circ} \mathrm{C}, 45-55 \%\right.$ relative humidity) for $0-120 \mathrm{~h}$. The virus remaining on the surface was then collected in $1.0 \mathrm{~mL}$ of DMEM and titrated (6). The detection limit for the titer of the virus remaining on the surface was $10^{0.5}$ TCID 50 . Survival time was defined as the time until the virus on the surface was no longer detected. Three independent experiments were performed for each condition, and the results of residual virus titers on the surfaces were expressed as the mean \pm standard error of the mean.

\section{Evaluation of effectiveness of alcohol-based disinfectants}


The effectiveness of the ethanol-based disinfectants was evaluated at different concentrations. The effectiveness of ethanol (EA, Nacalai Tesque) was tested at concentrations of $80 \%, 60 \%$, $50 \%, 40 \%, 35 \%, 32.5 \%, 30 \%, 27.5 \%, 25 \%, 22.5 \%$ and $20 \%(w / w)$. Isopropanol (IPA, Nacalai Tesque) was tested at a concentration of $70 \%(\mathrm{w} / \mathrm{w})$.

First, an in vitro evaluation of the effectiveness of disinfectant was performed. In a 1500 $\mu \mathrm{L}$ tube; $5 \mu \mathrm{L}$ of PBS containing virus $\left(5.0 \times 10^{4} \mathrm{TCID}_{50}\right.$ in $5 \mu \mathrm{L}$ PBS $)$ was mixed with $45 \mu \mathrm{L}$ of various disinfectants for $15 \mathrm{~s}$. Subsequently, the resulting solutions were neutralized with $450 \mu \mathrm{L}$ of DMEM, and the remaining viral titers were measured (14). The detection limit for the virus titers was $10^{0.2}$ TCID $_{50}$.

Next, the effectiveness of disinfectants against viruses on human skin was evaluated using the constructed model (ex vivo evaluation). Each virus solution $\left(1.0 \times 10^{5} \mathrm{TCID}_{50}\right.$ in $2 \mu \mathrm{L}$ PBS) was applied to the human skin surface. Each skin sample was then incubated for 15 min at $25^{\circ} \mathrm{C}$ with $45 \%-55 \%$ relative humidity to completely dry the viral mixture on the skin.

Subsequently, $18 \mu \mathrm{L}$ of disinfectant was applied to each skin sample surface for $15 \mathrm{~s}$ and then air-dried for $5 \mathrm{~min}$. After drying, the remaining viruses on the skin were recovered with $1000 \mu \mathrm{L}$ of DMEM, and the remaining viral load was measured (14). The detection limit for the virus titers was $10^{0.5} \mathrm{TCID}_{50}$. To determine the effectiveness of the disinfectants under each condition, logarithmic reductions in virus titers were calculated, normalizing to the PBS control titers. Three independent experiments were performed for each condition, and the results were expressed as the mean \pm standard error of the mean.

\section{Ethical considerations}


The study protocol, including sample collection procedures, was reviewed and approved by the Institutional Review Board of the Kyoto Prefectural University of Medicine (ERB-C-1593). Written informed consent was obtained from all the study participants.

\section{Statistical analysis}

Data were analyzed using GraphPad Prism 7 (GraphPad, Inc., La Jolla, CA, USA). The elapsed time was defined as an explanatory variable (X-axis), and the log virus titer was defined as an explained variable (Y-axis). Linear regression analysis with a logarithmic link function was performed to create a regression curve. The measurement limit of the SARS-CoV-2 titer was $10^{0.5} \mathrm{TCID}_{50}$; therefore, the survival time was defined as the X-value when the Y-values of the regression curves were 0.5 . The half-life was calculated from the slope of each regression curve when the titers of virus remaining on the surface were 2.0 and $3.0 \log _{10} \operatorname{TCID}_{50}(6,12)$. 


\section{Results}

The virus titers remaining on the plastic or human skin surfaces were measured over time (Supplementary Figure S1), and the survival time and half-life were calculated from these titer values by regression analysis (Supplementary Figure S2). In the plastic surface analysis, survival times of the Wuhan strain, Alpha variant, Beta variant, Gamma variant, Delta variant, and Omicron variant were 56.0 h (95\% confidence interval [CI], 39.0-76.7 h), $191.3 \mathrm{~h}(95 \% \mathrm{CI}$, 152.5-232.1 h), 156.6 h (95\% CI, 122.7-192.9 h), 59.3 h (95\% CI, 43.9-77.7 h), 114.0 h (95\% CI, 91.3-139.1 h), and 193.5 h (95\% CI, 153.1-236.2 h), respectively (Figure 1A and Table 1). In the human skin surface analysis, survival times of the Wuhan strain, Alpha variant, Beta variant, Gamma variant, Delta variant, and Omicron variant were 8.6 h (95\% CI, 6.5-10.9 h), 19.6 h (95\% CI, 14.8-25.3 h), 19.1 h (95\% CI, 13.9-25.3 h), 11.0 h (95\% CI, 8.1-14.7 h), 16.8 h (95\% CI, 13.1-21.1 h), and 21.1 h (95\% CI, 15.8-27.6 h), respectively (Figure 1B and Table 1). Alpha, Beta, Delta, and Omicron variants had significantly longer survival times than the Wuhan strain, and the Omicron variant had the longest survival time. Furthermore, the half-life showed the same tendency as the survival time (Figure 1C and 1D and Table 2).

The in vitro disinfectant effectiveness evaluation showed that the Wuhan strain and Gamma variant were completely inactivated within $15 \mathrm{~s}$ by $32.5 \%$ EA ( $\log$ reduction $>4)$, Alpha, Beta, and Delta variants were completely inactivated within $15 \mathrm{~s}$ by $35 \%$ EA, and the Omicron variant was completely inactivated within $15 \mathrm{~s}$ by $40 \%$ EA (Figure $2 \mathrm{~A}$ and Supplementary Table S1). Alpha, Beta, Delta, and Omicron variants were thus slightly more resistant to ethanol than the Wuhan strain. However, on human skin, an ex vivo evaluation showed that all viruses were completely inactivated after exposure to $35 \%$ EA for $15 \mathrm{~s}$ ( $\log$ reduction $>4$; Figure $2 \mathrm{~B}$ and Supplementary Table S2). 


\section{Discussion}

In 2020, the environmental stability of the Wuhan strain was reported in several studies $(6,7$, 15). Additionally, some studies suggested that the Alpha and Beta variants have the same degree of environmental stability $(8,9)$. However, no study has directly compared other VOCs with the Wuhan strain, and the differences in environmental stability between the Wuhan strain and all VOCs, including Omicron and Delta variants, were previously unknown.

Our study showed that on plastic and skin surfaces, Alpha, Beta, Delta, and Omicron variants exhibited more than two-fold longer survival times than those of the Wuhan strain and maintained infectivity for more than $16 \mathrm{~h}$ on the skin surfaces. The high environmental stability of these VOCs could increase the risk of contact transmission and contribute to the spread of VOCs. Additionally, in this analysis, there was no significant difference in survival times between Alpha and Beta variants, and they had similar environmental stability, which is consistent with the results of previous studies $(8,9)$.

The Omicron variant is currently a major concern owing to the rapidly increasing number of infected patients worldwide. The shift in the target site of infection from the lower respiratory tract to the upper respiratory tract and the escape from neutralizing antibodies might be potential factors for the spread of the Omicron variant (1-5). This study showed that the Omicron variant also has the highest environmental stability among VOCs, which suggests that this high stability might also be one of the factors that have allowed the Omicron variant to replace the Delta variant and spread rapidly. Although Alpha, Beta, Delta, and Omicron variants showed a slight increase in ethanol resistance in response to increased environmental stability, all VOCs on the skin surface were completely inactivated by $15 \mathrm{~s}$ exposure to $35 \%$ EA. Therefore, it is highly recommended that current infection control (hand hygiene) practices use 
disinfectants with appropriate EA concentrations ( $>52 \mathrm{w} / \mathrm{w} \%$ or $>60 \mathrm{v} / \mathrm{v} \%$ ), as proposed by the World Health Organization (16, 17).

This study had three limitations. First, the reason for the higher environmental stability of Alpha, Beta, Delta, and Omicron variants is unknown at this stage, and evaluation using recombinant viruses might identify factors that determine this. Second, the survival time and half-life obtained in this environmental stability evaluation might vary depending on the external environment and the composition of the body fluid containing the virus. In this study, to accurately analyze the differences in stability between VOCs, the target virus was purified by ultracentrifugation, and PBS was used as a solvent. Third, the relationship between the amount of virus remaining on the surface and the risk of transmission is still unclear at this stage. Therefore, it might be reasonable to interpret the value of survival time in this study as a reference value.

In conclusion, we elucidated the environmental stability of VOCs, which is important information for infection control. Furthermore, these findings will contribute greatly to elucidating the mechanism of VOC spread with the addition of genetic analysis. 


\section{Acknowledgments}

We thank Editage (www.editage.com) for English language editing. This research was supported by Adaptable and Seamless Technology Transfer Program through Target-driven R\&D (ASTEP) from the Japan Science and Technology Agency (JST) [grant number JPMJTR21UE and JPMJTM20PR], JSPS KAKENHI (grant number 21K16326), Mitsubishi Foundation, and Takeda Science Foundation.

\section{Author contributions}

Study concept and design: RH. Data acquisition: RH, HM, NW, TY, RB, TD. Data analysis and interpretation: RH, YI, and TN. Drafting of the manuscript: RH. Statistical analysis: RH. Secured funding: RH. Administrative/technical/material support: RH, HI. Study supervision: RH and TN.

\section{Competing financial interests}

The authors declare no competing financial interests. 


\section{References}

1. Tracking SARS-CoV-2 variants: World Health Organization; 2021 [Available from: https://www.who.int/en/activities/tracking-SARS-CoV-2-variants/.

2. SARS-CoV-2 B.1.1.529 (Omicron) Variant - United States, December 1-8, 2021. MMWR Morbidity and mortality weekly report. 2021;70(50):1731-4.

3. National Center for I, Respiratory Diseases DoVD. Science Brief: Omicron (B.1.1.529)

Variant. CDC COVID-19 Science Briefs. Atlanta (GA): Centers for Disease Control and Prevention (US)

Unless a copyright is indicated, information on CDC's sites, blogs, and applications is in the public domain and may be copied and distributed without permission.; 2020.

4. Enhancing readiness for Omicron (B.1.1.529): Technical brief and priority actions for Member States. World Health Organization: World Health Organization; 2021 [Available from: https://www.who.int/docs/default-source/coronaviruse/2021-12-23-global-technical-brief-andpriority-action-on-omicron.pdf?sfvrsn=d0e9fb6c_8.

5. He X, Hong W, Pan X, Lu G, Wei X. SARS-CoV-2 Omicron variant: Characteristics and prevention. MedComm. 2021;2(4):838-45.

6. Hirose R, Ikegaya H, Naito Y, Watanabe N, Yoshida T, Bandou R, et al. Survival of SARS-CoV-2 and influenza virus on the human skin: Importance of hand hygiene in COVID-19. Clinical infectious diseases : an official publication of the Infectious Diseases Society of America. 2020.

7. van Doremalen N, Bushmaker T, Morris DH, Holbrook MG, Gamble A, Williamson BN, et al. Aerosol and Surface Stability of SARS-CoV-2 as Compared with SARS-CoV-1. The New England journal of medicine. 2020;382(16):1564-7. 
8. Pottage T, Garratt I, Onianwa O, Spencer A, Paton S, Verlander NQ, et al. A comparison of persistence of SARS-CoV-2 variants on stainless steel. The Journal of hospital infection. 2021;114:163-6.

9. Meister TL, Fortmann J, Todt D, Heinen N, Ludwig A, Brüggemann Y, et al. Comparable Environmental Stability and Disinfection Profiles of the Currently Circulating SARS-CoV-2 Variants of Concern B.1.1.7 and B.1.351. The Journal of infectious diseases. $2021 ; 224(3): 420-4$.

10. Matsuyama S, Nao N, Shirato K, Kawase M, Saito S, Takayama I, et al. Enhanced isolation of SARS-CoV-2 by TMPRSS2-expressing cells. Proceedings of the National Academy of Sciences of the United States of America. 2020;117(13):7001-3.

11. Matsuyama S, Nagata N, Shirato K, Kawase M, Takeda M, Taguchi F. Efficient activation of the severe acute respiratory syndrome coronavirus spike protein by the transmembrane protease TMPRSS2. Journal of virology. 2010;84(24):12658-64.

12. Hirose R, Itoh Y, Ikegaya H, Miyazaki H, Watanabe N, Yoshida T, et al. Evaluation of the Residual Disinfection Effects of Commonly Used Skin Disinfectants against Viruses: An Innovative Contact Transmission Control Method. Environmental Science \& Technology. $2021 ; 55(23): 16044-55$.

13. Barcena M, Oostergetel GT, Bartelink W, Faas FG, Verkleij A, Rottier PJ, et al. Cryoelectron tomography of mouse hepatitis virus: Insights into the structure of the coronavirion. Proceedings of the National Academy of Sciences of the United States of America. $2009 ; 106(2): 582-7$

14. Hirose R, Bandou R, Ikegaya H, Watanabe N, Yoshida T, Daidoji T, et al. Disinfectant effectiveness against SARS-CoV-2 and influenza viruses present on human skin: model-based 
evaluation. Clinical microbiology and infection : the official publication of the European Society of Clinical Microbiology and Infectious Diseases. 2021.

15. Chin AWH, Chu JTS, Perera MRA, Hui KPY, Yen HL, Chan MCW, et al. Stability of SARS-CoV-2 in different environmental conditions. The Lancet Microbe. 2020;1(1):e10.

16. Golin AP, Choi D, Ghahary A. Hand sanitizers: A review of ingredients, mechanisms of action, modes of delivery, and efficacy against coronaviruses. American journal of infection control. 2020;48(9):1062-7.

17. Infection prevention and control during health care when novel coronavirus (nCoV) infection is suspected. World Health Organization.: World Health Organization; 2020 [Available from: https://www.who.int/publications/i/item/10665-331495. 
Table 1. Survival time of SARS-CoV-2 on plastic and human skin surfaces.

\begin{tabular}{lcc}
\hline & \multicolumn{2}{c}{ Survival time, hour, median $(95 \% \mathrm{CI})$} \\
\cline { 2 - 3 } Wuhan strain & $56.0(39.0-76.7)$ & Skin surface \\
\hline Alpha variant & $191.3(152.5-232.1)$ & $8.6(6.5-10.9)$ \\
Beta variant & $156.6(122.7-192.9)$ & $19.6(14.8-25.3)$ \\
Gamma variant & $59.3(43.9-77.7)$ & $19.1(13.9-25.3)$ \\
Delta variant & $114.0(91.3-139.1)$ & $11.0(8.1-14.7)$ \\
Omicron variant & $193.5(153.1-236.2)$ & $21.1(15.8-27.6)$ \\
\hline
\end{tabular}

The elapsed time was defined as an explanatory variable (X-axis), and the log virus titer was defined as an explained variable (Y-axis). A linear regression analysis with logarithmic link function was performed to create a curve of regression. The measurement limit of the SARSCoV-2 titer was $10^{0.5} \mathrm{TCID}_{50}$; therefore, the survival time was defined as the $\mathrm{X}$ values when the $\mathrm{Y}$ values of the regression curves were 0.5 . 
Table 2. Half-life of SARS-CoV-2 on the plastic and human skin surfaces.

\begin{tabular}{lcccc}
\hline & \multicolumn{4}{c}{ Half-life, hour, median $(95 \% \mathrm{CI})$} \\
\cline { 2 - 5 } & \multicolumn{2}{c}{ Plastic surface } & \multicolumn{2}{c}{ Skin surface } \\
\cline { 2 - 5 } & $\mathbf{3}\left(\log _{10} \mathrm{TCID}_{50}\right)$ & $\mathbf{2}\left(\log _{10} \mathrm{TCID}_{50}\right)$ & $\mathbf{3}\left(\log _{10} \mathrm{TCID}_{50}\right)$ & $\mathbf{2}\left(\log _{10} \mathrm{TCID}_{50}\right)$ \\
\hline Wuhan strain & $3.5(2.0-5.7)$ & $5.2(3.0-8.6)$ & $0.5(0.3-0.7)$ & $0.7(0.5-1.1)$ \\
Alpha variant & $9.9(7.9-12.7)$ & $14.9(11.9-19.1)$ & $1.1(0.8-1.6)$ & $1.7(1.2-2.4)$ \\
Beta variant & $8.3(6.4-10.9)$ & $12.5(9.7-16.3)$ & $1.2(0.8-1.8)$ & $1.7(1.2-2.6)$ \\
Gamma variant & $3.9(2.5-5.8)$ & $5.8(3.7-8.7)$ & $0.7(0.5-1.1)$ & $1.1(0.7-1.7)$ \\
Delta variant & $6.7(5.1-8.7)$ & $10.1(7.7-13.1)$ & $1.0(0.8-1.4)$ & $1.5(1.1-2.1)$ \\
Omicron variant & $10.0(8.0-12.9)$ & $15.1(11.9-19.4)$ & $1.4(1.0-2.0)$ & $2.0(1.4-3.0)$ \\
\hline
\end{tabular}

The elapsed time was defined as an explanatory variable (X-axis), and the log virus titer was defined as an explained variable (Y-axis). A linear regression analysis with logarithmic link function was performed to create a curve of regression. The half-life was calculated from the slope of each regression curve when the titer of virus remaining on the surface was 2.0 , and 3.0 $\log _{10} \mathrm{TCID}_{50}$. 

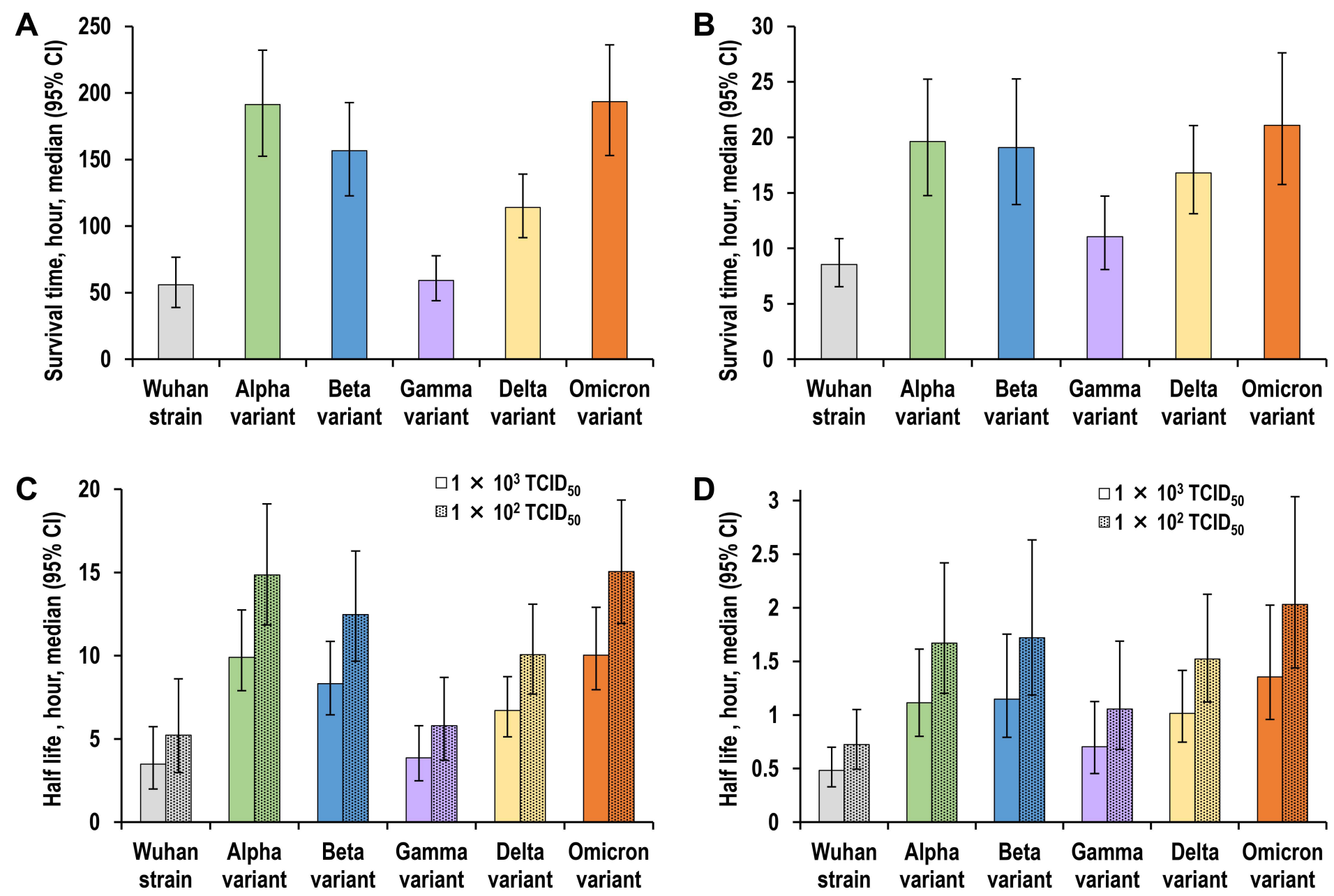

Figure 1. (A) Survival times of the various viruses on a plastic surface. (B) Survival times of the various viruses on the surface of human skin. (C) Half-lives of the various viruses on a plastic surface. (D) Half-lives of the various viruses on the surface of human skin. Survival time is defined as the time until the virus was no longer detected on the surface. All half-lives in the graphs refer to a condition when $1 \times 10^{2}$ or $1 \times 10^{3} \mathrm{TCID}_{50}(50 \%$ tissue culture infectious dose) of virus particles remain on the surface. Data are expressed as the median $\pm 95 \%$ confidence interval. 

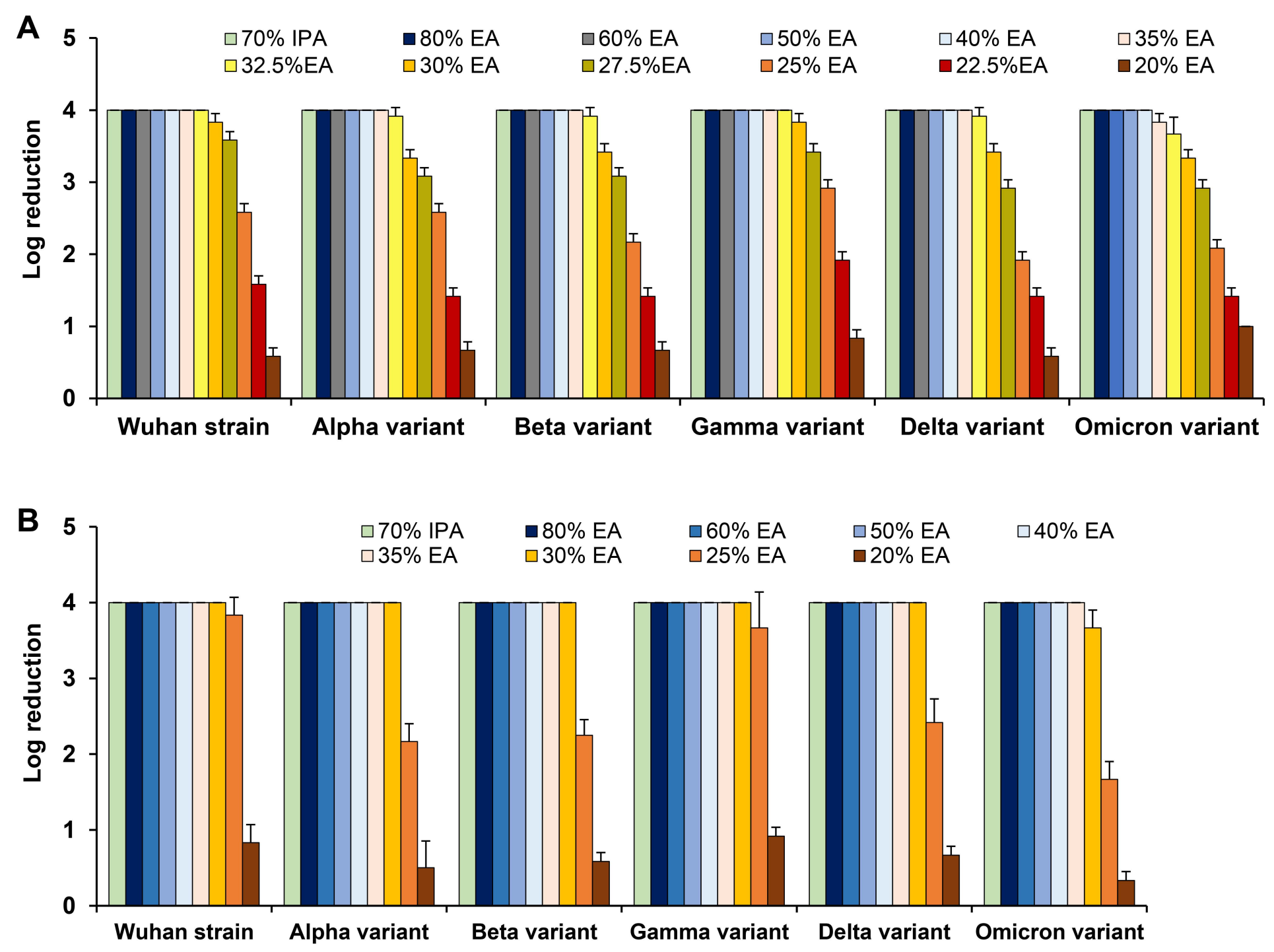

Figure 2. Evaluation of disinfectant effectiveness. Both in vitro evaluation (A) and ex vivo evaluation (B) were performed, and the log reduction was calculated from the residual viral titer after each alcohol-based disinfectant exposure (see Supplementary Table S1 and S2). The results are expressed as the mean \pm standard error. EA, ethyl alcohol; IPA, isopropanol. 